\begin{tabular}{l|l} 
REVISTA & $\begin{array}{l}\text { Revista Educación } \\
\text { ISSN: 0379-7082 } \\
\text { ISSN: 2215-2644 } \\
\text { revedu@gmail.com } \\
\text { Universidad de Costa Rica } \\
\text { Costa Rica }\end{array}$
\end{tabular}

\title{
Investigación y formación: un diálogo necesario
}

González Campos, José Alejandro; Medina Pérez, Jessica

Investigación y formación: un diálogo necesario

Revista Educación, vol. 46, núm. 1, 2022

Universidad de Costa Rica, Costa Rica

Disponible en: https://www.redalyc.org/articulo.oa?id=44068165035

DOI: https://doi.org/10.15517/revedu.v46i1.45411

\section{(c) 1 (1)}

Esta obra está bajo una Licencia Creative Commons Atribución-NoComercial-SinDerivar 3.0 Internacional. 
Artículos Científicos

\title{
Investigación y formación: un diálogo necesario
}

\author{
Research and Training: A Necessary Dialogue \\ José Alejandro González Campos \\ Universidad de Playa Ancha, Chile \\ jgonzalez@upla.cl \\ (iD) https://orcid.org/0000-0003-4610-6874 \\ Jessica Medina Pérez \\ Pontificia Universidad Católica de Valparaiso, Chile \\ jessica.medina@pucv.cl \\ (iD https://orcid.org/0000-0003-2721-9929
}
DOI: https://doi.org/10.15517/revedu.v46i1.45411 Redalyc: https://www.redalyc.org/articulo.oa? $\mathrm{id}=44068165035$

Recepción: 09 Febrero 2021

Aprobación: 25 Mayo 2021

\section{Resumen:}

La investigación debe contribuir a la formación de profesionales y no solo a requisitos de acreditación. Por ello, este estudio de tipo cuantitativo deja en evidencia, mediante el levantamiento de dos índices denominados de vigencia y DIAFORIN (Diálogo entre Formación e Investigación), el bajo nivel de impacto de la investigación dentro de sus procesos formativos (programas de estudio de las carreras de pedagogía de una universidad regional y estatal de Chile). Para la operacionalización de los índices se utilizaron 124 programas formativos, que enmarcan este estudio en un paradigma positivista, cuyo nivel de profundización es inferencial. Entre las variables que se consideraron para la construcción de los índices se encuentran el número de referencias, número de referencias en inglés, año de la referencia más reciente, número de referencias por lo menos con una persona autora de la universidad, entre otras. Los valores observados de ambos índices (vigencia y DIAFORIN) dan evidencia de grandes desafíos, como la consolidación del diálogo entre la investigación y la formación en educación superior; adicionalmente, contribuye a promover planes formativos actualizados que integran conceptos como internacionalización y conocimiento dinámico.

Palabras Clave: Programas formativos, Internacionalización, Formación inicial docente, Aporte de la investigación.

\section{ABstraCT:}

Since research should contribute to training professionals and not only to accreditation requirements, a quantitative study was conducted to demonstrate how developing two indexes, the Validity and DIAFORIN (Dialogue between Training and Research) serves to reflect the low impact of research on training programs (study programs for education majors at a regional and state university in Chile). The aforementioned indexes are used for 124 training programs to serve as a framework for the study using a positivist paradigm with inferential depth. Variables considered for elaborating these indexes include the number of references in English, the year of the most recent reference, number of references with at least one author from the institutions, among others. The observed values of both the Validity and DIAFORIN indexes reflect the challenge of juxtaposing research and training in higher education which, also, contribute to promoting updated training plans that integrate concepts such as internationalization and knowledge.

KEYWORDS: Education Programs, Internationalization, Preservice Teacher, Research Contribution.

\section{INTRODUCCIÓN}

La investigación, sin duda, contribuye no solo a enriquecer la formación de las personas profesionales, sino que ayuda a mejorar diversas prácticas durante la formación de profesionales que incidirán luego en la vida laboral; sin embargo, en los últimos años, quizás por los requisitos de la acreditación, la investigación no necesariamente ha contribuido con este propósito y se ha transformado en solo un ranking que debe alcanzarse (Martí et al., 2018). 
Por ello, en una primera parte este estudio detalla la importancia de la investigación y cómo esta debe ser un aporte en la educación superior, pero principalmente enfatiza que debe ser parte de la formación inicial docente, ya que es el camino a seguir para mejorar las futuras prácticas de aprendizaje-enseñanza. Por otro lado, se evidencia la importancia de los programas de estudio como guía para lograr el perfil de egreso declarado por la institución.

En una segunda etapa, se describe la metodología con sus respectivos objetivos, hipótesis, universo y la propuesta métrica de los índices de vigencia y DIAFORIN.

Finalmente, se concluye cómo los índices en investigación son una medida útil para comprender y resumir un conjunto de datos referidos al impacto por la cantidad de citas, la rigurosidad del proceso de evaluación y la ausencia de índices que expliquen el impacto de la investigación en los programas de estudio y, posteriormente, en la formación del estudiantado.

Dado lo anterior, surge el siguiente problema de investigación: ¿Se encuentra presente la investigación que realiza la universidad en los programas de estudios de las carreras de pedagogía?, ¿qué tan actualizados son los programas de estudio de estas carreras? y ¿cuál es el grado de presencia de la investigación producida por la universidad en estos programas?

Para responder estas preguntas se ha planteado como objetivo: definir y caracterizar un indice que cuantifique y explique el grado de asociación entre la formación y la investigación, evidenciada en los planes formativos de las carreras pedagógicas, y se construyeron los índices de vigencia y DIAFORIN que midieron diversas variables de la composición de estos programas; se aplicaron a 124 programas de estudio de las carreras de pedagogía de una universidad regional y estatal de Chile, donde se evidenció el bajo aporte de investigación de la universidad y una escasa actualización de los programas de estudios formativos.

\section{MARCo TEÓRICo}

\subsection{Investigación y docencia}

Una de las grandes contribuciones de la academia, principalmente de las universidades, es la investigación. Esta ha sido utilizada para generar nuevos conocimientos y potenciar los existentes, pero, sobre todo, debe ser un aporte al estudiantado de las instituciones, que contribuya a la adquisición de nuevos conocimientos, destrezas y especialmente al desarrollo de nuevas personas profesionales (Erraéz-Alvarado et al., 2020). Para Restrepo (2003), la investigación, especialmente la investigación en el ámbito de la pedagogía, es fundamental tanto para la formación del futuro profesorado, como durante su desarrollo laboral, ya que constantemente debe tomar las evidencias de su práctica para mejorar su trabajo con base en los resultados obtenidos, con lo que logra alcanzar aprendizajes más significativos en sus estudiantes.

Tejeda (2015) definió el aporte teórico de la investigación a la pedagogía como el conocimiento que se relaciona con los problemas y realidad educativa actual. Este aporte debe tener: (1) pertinencia: posibilidad real de aplicación, (2) novedad: oportunidad de mejorar el conocimiento existente, (3) validez: soluciona parte o todo de un problema existente y (4) coherencia científica: contiene los fundamentos epistemológicos de la educación. Este mismo autor describe al proceso educativo vinculado a la investigación, por lo que deben responder a necesidades y problemas prácticos, es decir, utilizar las nuevas investigaciones constantemente como contribución y solución a las nuevas necesidades (Tejeda, 2015).

Una de las estrategias implementadas por las universidades para potenciar la investigación en el estudiantado, tanto de pregrado como de posgrado han sido los semilleros de investigación, con ellos han mejorado sus indicadores de investigación e innovación realizada tanto por estudiantes como por profesores y profesoras, aportando de esta manera, con nuevo conocimiento en diversas áreas de estudio (Quintero et al., 2008). Rodríguez et al. (2019), en su artículo publicado en el libro Innovación Educativa en la Sociedad 
Digital destaca la necesidad de la formación en investigación como parte de todo el proceso de formación de cualquier profesional, ya que se aprende a investigar investigando y no como parte de un solo curso o asignatura; en la propuesta destacan a la iniciativa semilleros de investigación como una contribución a la formación de profesionales y al aumento del interés por continuar estudios de posgrado.

Diversos estudios, como el de Martí et al. (2018), describen el problema de la investigación en la actualidad solo como parte de mecanismos de acreditación y rankings que se deben alcanzar y se encuentra lejos de ser un aporte para la solución de problemas reales y, más aún, una contribución a la formación de sus propios estudiantes. Para ello, estas personas autoras, basadas en un informe de la UNESCO (2015), consideran a la educación superior como un bien común y proponen como solución a este problema un rediseño en la toma de decisiones y mecanismos de gobernanza de las universidades, en la que deben mirar a la investigación universitaria como un servicio a la sociedad y parte de la formación de sus estudiantes (UNESCO, 2015; Martí et al., 2018), de lo contrario, la investigación perderá su sentido, al igual que el tiempo y los recursos invertidos cuando no es posible utilizarla para mejorar los problemas actuales (Perines, 2017).

En los años 90 ya se evidenciaban dificultades en la investigación, donde se hacía notar el poco uso práctico de esta, lo que la llevó a un desprestigio (Perines, 2018). Diversos estudios, como el Perines (2017), proponen enfatizar y movilizar el conocimiento científico, específicamente la investigación que produce cada universidad, a través de capacitaciones específicas y jornadas de intercambios, adicionalmente, actualizar de manera constante los programas de estudios, para así otorgar la oportunidad de la incorporación del nuevo conocimiento. No obstante, el trabajo no finaliza cuando la investigación es conocida por la comunidad docente y estudiantil, sino que debe existir una conexión y bajada al trabajo práctico apoyada por las instituciones, al mismo tiempo los programas de estudio deben preparar a las futuras y futuros docentes en investigación e incluir el aporte del profesorado de la institución en las actualizaciones, es así como Perines y Campaña (2019) propone cuatro formas de integración: (1) investigación presente en programas de asignaturas y perfil de egreso, (2) docentes con un rol investigador, (3) uso de artículos en clases e (4) incluir a futuras personas docentes en proyectos de investigación.

\subsection{Integración de la investigación en los programas formativos de pedagogía}

En distintos lugares del mundo, Chile incluido, las universidades confeccionan sus propios programas de estudio (Grossman y McDonald, 2008; Perines 2018), lo que repercute en que cada institución prioriza distintas habilidades y criterios, esto lleva a encontrar algunos muy completos e innovadores y otros con características más débiles (Perines, 2018).

En los últimos años los estudios sobre cómo construir un programa de estudio en educación superior se han incrementado, un ejemplo de ello es el trabajo de Díaz (2014), en el que propone construir los programas con base en las competencias que se busca desarrollar, con apoyo en verbos e indicadores de desempeño para guiar esta elaboración; sin embargo, no existen lineamientos claros sobre el tipo de documentos, investigaciones o bibliografía en la que deberían basarse para su construcción y, finalmente cómo deberían ser las referencias bibliográficas que se deben encontrar en un programa de estudio (Díaz, 2014). Asimismo, no existen evidencias o investigaciones de cómo la investigación que producen las universidades contribuye al conocimiento y construcción de estos programas de estudio (Martí et al., 2018).

Otras investigaciones manifiestan la urgente necesidad de establecer lineamientos claros para la construcción de programas de estudios, ya que no es posible visualizar el enfoque por competencias que debe desarrollarse en la actualidad y no existen acuerdos en cómo se debe diseñar un programa de estudios basado en competencias (Merterns, 1997). Sin embargo, muchas personas expertas coinciden en que los programas para las carreras de pedagogía deben estar compuestos por competencias profesionales o pedagógicas, transversales y específicas de la disciplina (Aneas y Vila, 2018). Es así como Balladares-Burgos (2018) realiza una propuesta con cuatro categorías clave para la elaboración de programas de pedagogía en modalidad 
virtual: (1) competencias específicas, (2) innovación, (3) investigación y (4) diseño de actividades. De esta manera, la persona autora contribuye con una propuesta para diseños de programas actualizados para la formación de nuevo profesorado, con nuevas competencias y adaptado a los desafíos educativos actuales (Balladares-Burgos, 2018).

Las universidades cubanas, las facultades de Ciencias Médicas específicamente, analizan constantemente sus programas de estudio como parte de la evaluación de sus docentes, es decir, cada profesor y profesora tiene la responsabilidad de revisar, analizar y evaluar el programa de su asignatura como parte de sus tareas; algunos de los aspectos que consideran para dicha evaluación son: las unidades, tiempo destinado a cada una de las unidades, habilidades declaradas, nivel de profundidad de las habilidades, los tipos de evaluaciones y, finalmente, la bibliografía, en la que se visualizan aspectos como la pertinencia y accesibilidad de cada una de las citas, si poseen bibliografía básica, complementaria o multimedia (Quintana et al., 2013). Sin embargo, no evalúan aspectos como el aporte en investigación de la universidad o con cuánta bibliografía actualizada cuentan.

En la actualidad, la construcción de los programas de estudio se basa en dos grandes y nuevos desafíos, estos son la perspectiva de género (Camarena y Saavedra, 2018) y la virtualidad (Briceño et al., 2020). En aspectos relacionados al género, se deben incorporar los estudios que existen en la materia, donde se reconoce que mujeres y hombres tienen diferentes enfoques y estilos de liderazgos, y desde ahí fomentar su equidad (Camarena y Saavedra, 2018). Para el caso de la virtualidad, se deben considerar las exigencias de la internacionalización con nuevos diseños instruccionales focalizados en el apoyo tecnológico, una nueva gestión académica de soporte y una cultura de evaluación con altos estándares de calidad (Briceño et al., 2020).

\section{3. Índices en educación superior}

Uno de los grandes desafíos de la investigación es establecer cómo se medirá lo que se investigará. Como respuesta a esta problemática, distintas personas autoras, como Boudon y Lazarsfeld (1973), proponen levantar índices. Por tanto, un índice es definido como una medida única levantada con base en la información recolectada, esto ayuda a resumir un conjunto de indicadores y las relaciones entre ellos para, finalmente, determinar su peso o influencia (Boudon y Lazarsfeld, 1973).

Los índices son un tipo de medida con la que se han realizado estudios en Ciencias Sociales, un ejemplo de ello es el utilizado en área de Educación por Medina y González (2020), en el que proponen un índice estocástico de similaridad para medir el logro del perfil de egreso del estudiantado de Pedagogía, de esta manera establecen qué tan parecido es el logro del perfil año a año en relación a un perfil final o integral. Sin embargo, no es posible encontrar estudios sobre un índice que explique el impacto de la investigación de la misma institución formadora u otra en la formación de profesoras y profesores.

Es importante señalar que contar con un índice, en cualquier área de estudio, trae importantes ventajas como: conocer valores esperados o esperanza, determinar factores de más peso y, en general, contar con mayor información para la toma de decisiones (Medina y González, 2020).

\section{Metodología}

\subsection{Tipo y alcance de la investigación}

Esta investigación se enmarca en un paradigma positivista, de tipo cuantitativo, cuyo nivel de profundización es inferencial, con registro prospectivo, transversal y no experimental. 


\subsection{Objetivo}

En la investigación se ha planteado el siguiente objetivo general: definir y caracterizar un índice que cuantifique y explique el grado de asociación entre la formación y la investigación, evidenciada en los planes formativos de las carreras pedagógicas.

Adicionalmente, se han planteado los siguientes objetivos específicos: (1) definir y describir indicadores continuos para resumir componentes nucleares de los planes formativos a la luz de las nuevas exigencias formativas en educación superior; (2) jerarquizar los indicadores definidos en función de la prioridad identificada en los planes formativos; (3) definir categorías de consistencia en función del comportamiento de los indicadores e índices.

\subsection{Preguntas guía de investigación}

En consideración de definir y caracterizar un índice que describa la consistencia entre la formación y la investigación, es que el interés investigativo se orienta por estas preguntas: (1) ¿En qué grado los planes formativos de las carreras pedagógicas consideran o incluyen como parte de sus referentes, los resultados de investigación de sus propios investigadores e investigadoras? (2) ¿Qué atributo o característica métrica, en función de las variables de estudio, es la de mayor frecuencia en los planes formativos de las carreras pedagógicas? (3) ¿Existe algún estándar para la presencia y grado de las variables de estudio en los planes formativos para las carreras pedagógicas?

\subsection{Variables y su operacionalización}

Para la especificación del estatus métrico y la operacionalización de las variables, se tomó en consideración la definición de dos índices denominados índice de vigencia e índice de Diálogo entre Formación e Investigación [DIAFORIN] (cuantificador del grado de asociación o diálogo entre la formación y la investigación traducida en las referencias bibliográficas de los planes formativos), en los cuales intervienen las siguientes variables: número de referencias, número de referencias en inglés, año de la referencia más antigua, año de la referencia más reciente, cuántas de las referencias son libros, artículos, tesis de la misma universidad, tesis que no son de la universidad en cuestión, documentos grises, documentos web y cuántas tienen, por lo menos, una persona autora de la universidad en cuestión. Todas las variables que se indican poseen un estatus métrico cuantitativo en escala de razón. Para el caso de los índices e indicadores en general, estos también poseen un estatus métrico cuantitativo en escala razón y, además, de soporte compacto en el intervalo $[0,1]$. Este soporte permite realizar conclusiones o interpretaciones en términos porcentuales, para facilitar la comunicación y comprensión de lo que se mide y qué tan próximo se está de lo que se quiere lograr.

\subsection{Instrumento o proceso de abstracción}

Para el proceso de abstracción de las variables de estudio se confeccionó un instrumento fijo (lista de cotejo) según objetivos. El proceso de abstracción fue revisado tres veces, para asegurar el correcto registro y conteo con un porcentaje de similitud superior al 95 \% (Medina y González, 2019). Los programas formativos se encuentran disponibles y de acceso abierto en la página web de la universidad. 


\subsection{Hipótesis de investigación}

En la investigación se levantaron, de acuerdo a los objetivos específicos, las siguientes preguntas guía de investigación e hipótesis de trabajo (Tabla 1).

TABLA 1.

Objetivos, preguntas de investigación e hipótesis.

\begin{tabular}{|c|c|c|}
\hline específicos & $\begin{array}{c}\text { Preguntas de } \\
\text { investigación guía }\end{array}$ & Hipótesis de trabajo \\
\hline $\begin{array}{l}\text { (1) Definir y } \text { describir } \\
\text { indicadores continuos } \\
\text { para resumir } \\
\text { componentes nucleares } \\
\text { de los planes formativos } \\
\text { a la luz de las nuevas } \\
\text { exigencias formativas en } \\
\text { educación superior. }\end{array}$ & $\begin{array}{l}\text { ¿En qué grado los planes } \\
\text { formativos de las } \\
\text { carreras pedagógicas } \\
\text { consideran o incluyen, } \\
\text { como parte de sus } \\
\text { referentes, los resultados } \\
\text { de investigación de sus } \\
\text { propios académicos y } \\
\text { académicas? }\end{array}$ & $\begin{array}{l}\text { El índice DIAFORIN es } \\
\text { significativamente mayor } \\
\text { que cero ( } \alpha=0.05) \text {, lo } \\
\text { que se traduce en una } \\
\text { dependencia significativa } \\
\text { entre lo que producen los } \\
\text { académicos } \\
\text { académicas en relación } \\
\text { con el conocimiento } \\
\text { científico y lo que es } \\
\text { parte de los programas } \\
\text { formativos. }\end{array}$ \\
\hline $\begin{array}{l}\text { (2) Jerarquizar los } \\
\text { indicadores definidos en } \\
\text { función de la prioridad } \\
\text { identificada en los planes } \\
\text { formativos. }\end{array}$ & $\begin{array}{l}\text { ¿Qué atributo o } \\
\text { característica métrica, en } \\
\text { función de las variables } \\
\text { de estudio, es la de } \\
\text { mayor frecuencia en los } \\
\text { planes formativos de las } \\
\text { carreras pedagógicas? }\end{array}$ & $\begin{array}{l}\text { El índice de conocimiento } \\
\text { estático es } \\
\text { significativamente mayor } \\
\text { que el indice de } \\
\text { conocimiento dinámico } \\
(\alpha=0.05) \text {. }\end{array}$ \\
\hline $\begin{array}{l}\text { (3) Definir categorías de } \\
\text { consistencia en función } \\
\text { del comportamiento de } \\
\text { los indicadores e índices. }\end{array}$ & $\begin{array}{l}\text { ¿Existe algún estándar } \\
\text { para la presencia y grado } \\
\text { de las variables de } \\
\text { estudio en los planes } \\
\text { formativos para las } \\
\text { carreras pedagógicas? }\end{array}$ & $\begin{array}{l}\text { No existe un estándar } \\
\text { referencial para guiar la } \\
\text { estructuración de un } \\
\text { programa formativo. }\end{array}$ \\
\hline
\end{tabular}

Fuente: elaboración propia. 


\subsection{Universo y muestra}

El universo de estudio corresponde a todos los programas formativos de las carreras pedagógicas de una Facultad de una universidad estatal chilena, correspondientes a lo disciplinar y a lo pedagógico. En el proceso de muestreo, prácticamente se incluyó a toda la población, salvo un par de programas que no contenían las referencias bibliográficas, lo que especifica un tamaño muestral de 124 programas formativos (el tamaño muestral es superior al establecido por la fórmula de Namakforoosh (2000), al considerar un nivel de confianza del $95 \%$, y error de estimación del $\pm 5 \%$.).

Los programas de estudio analizados, si bien tienen distintas características, tienen elementos en común que permiten su posterior análisis. Estos son: presencia de internacionalidad, actualidad o año, conocimiento dinámico, conocimiento estático, conocimiento gris, entre otros (ver Anexo 1).

\subsection{Proceso de análisis de datos}

Primero se realizó un análisis descriptivo para resumir las variables de estudio, en coherencia al estatus métrico de cada una de ellas. Posteriormente se realizaron pruebas inferenciales relativas a posibles valores para el parámetro de centralidad en los índices de vigencia y DIAFORIN. Posteriormente, se propone un modelo distribucional para los índices, donde se utilizan, como criterios de selección del modelo, los criterios AIC y BIC.

Los análisis estadísticos fueron realizados con el entorno de programación R 3.6.1 y Jamovi 1.2.27.

\subsection{Propuesta métrica de los índices de vigencia y DIAFORIN}

El índice de vigencia $\left(I_{v}\right)$ y el índice DIAFORIN $\left(I_{D}\right)$ son definidos como suma ponderada de cada uno de los siguientes indicadores: internacionalidad $(I I)$, actualidad $(I A)$, contribución universitaria (ICU, conocimiento dinámico (ICD), conocimiento estático $(I C E)$, conocimiento gris $(I C G)$. Las configuraciones funcionales para los índices de vigencia y DIAFORIN son:

$$
\begin{gathered}
I_{V}=\frac{(\mathrm{ICD} \cdot 0.45+\mathrm{ICE} \cdot 0.45+\mathrm{ICG} \cdot 0.1+\mathrm{II} \cdot 0.4+\mathrm{IA} \cdot 0.2+\mathrm{ICU} \cdot 0.2)}{2} \\
I_{D}=\mathrm{ICD} \cdot \mathrm{ICU}+\mathrm{ICE} \cdot \mathrm{ICU}
\end{gathered}
$$

Se observa que, según la definición de los índices, estos tienen un recorrido compacto, esto es: $I_{V} \in[0 ; 1]$ y $I_{D} \in[0 ; 1]$, lo que permite realizar interpretaciones en términos porcentuales (la especificación de cada indicador se presenta en el Anexo 1).

Definir estos índices en este intervalo compacto permite caracterizarlos como una variable aleatoria, cuyo mejor modelo (en función de su soporte y continuidad) es la distribución beta, que atribuye nuevas formas de interpretación en términos de probabilidades.

La forma funcional de una variable aleatoria con distribución beta de parámetros $\alpha$ y $\beta$ es dada por:

$$
\mathrm{f}(\mathrm{x})=\frac{\Gamma(\alpha+\beta)}{\Gamma(\alpha) \Gamma(\beta)} \mathrm{e}^{\alpha-1}(1-\mathrm{x})^{\beta-1} ; 0 \leq \mathrm{x} \leq 1
$$


El valor de x que constituye la fórmula antes descrita, representa el valor que puede ser observado para cada uno de los índices.

Disponer de un modelo ayuda a conocer el valor esperado o esperanza y la varianza, las cuales se representan

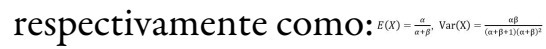

Es importante indicar que la estimación de los valores de $\alpha$ y $\beta$, permite explicitar completamente el modelo. Para la estimación se utilizará el método de máxima verosimilitud y, como criterio de selección de modelo, los criterios LogL, BIC y AIC.

Para la asociación a este modelo distribucional fue necesario realizar varios ajustes de diferentes modelos y diferentes simulaciones de datos, de donde se obtuvo que el modelo beta presenta los menores BIC y AIC (-271.3 y -276.83 respectivamente) y, en el caso del criterio LogL, la mayor puntuación (140.46), por tanto, se justifica su uso. En este caso los valores estimados para los parámetros son $\alpha=20 \mathrm{y} \beta=20$

Este modelo y los valores de los parámetros que serán utilizados como referencia para la toma de decisiones y la obtención de conclusiones, se denominarán como modelo estándar. Esto es: ¿qué tipo de asimetría se observa?, ¿qué tipo de curtosis?, ¿en cuánto incrementó o disminuyó la media?, entre otros cuestionamientos. Por otro lado, se soportan afirmaciones en probabilidad, por ejemplo, determinar las probabilidades de alcanzar la media del modelo estándar o determinar probabilidades de superar brechas preestablecidas (ver Tabla 2).

TABLA 2.

Momentos del modelo estándar.

\begin{tabular}{l|l}
\hline Momento & Valor \\
\hline Media & 0.5 \\
\hline Varianza & 0.0061 \\
\hline Simetría & 0 (Simétrica) \\
\hline Curtosis & 0.14 (Mesocurtica) \\
\hline
\end{tabular}

Fuente: Elaboración propia

\subsection{Procedimiento de Análisis}

1. Determinación de los índices de vigencia y DIAFORIN. Estos son obtenidos a partir de la muestra observada.

2. Determinación de la esperanza y varianza muestral.

3. Estimación de los parámetros $\alpha$ y $\beta$.

4. Representación gráfica en función de las estimaciones.

5. Comparación entre el modelo basado en la muestra observada y el modelo estándar.

6. Determinación de probabilidades y comparaciones en forma.

7. Generación de conclusiones.

\section{Resultados Y APLicación}

En el proceso de ajustar el modelo distribucional beta a los datos observados, se estimaron los parámetros del modelo en función de la muestra, donde se obtuvo que $\alpha=24$ y $\beta=45$. Este proceso de modelar consiste en tomar un modelo teórico de referencia, dada sus características y particularidades, y en función de la evidencia emanada de los datos para proponer un valor para cada parámetro, que haga que el modelo se ajuste a los datos. Este proceso es llamado estimación. 


\section{Comparación Observado-Estándar}

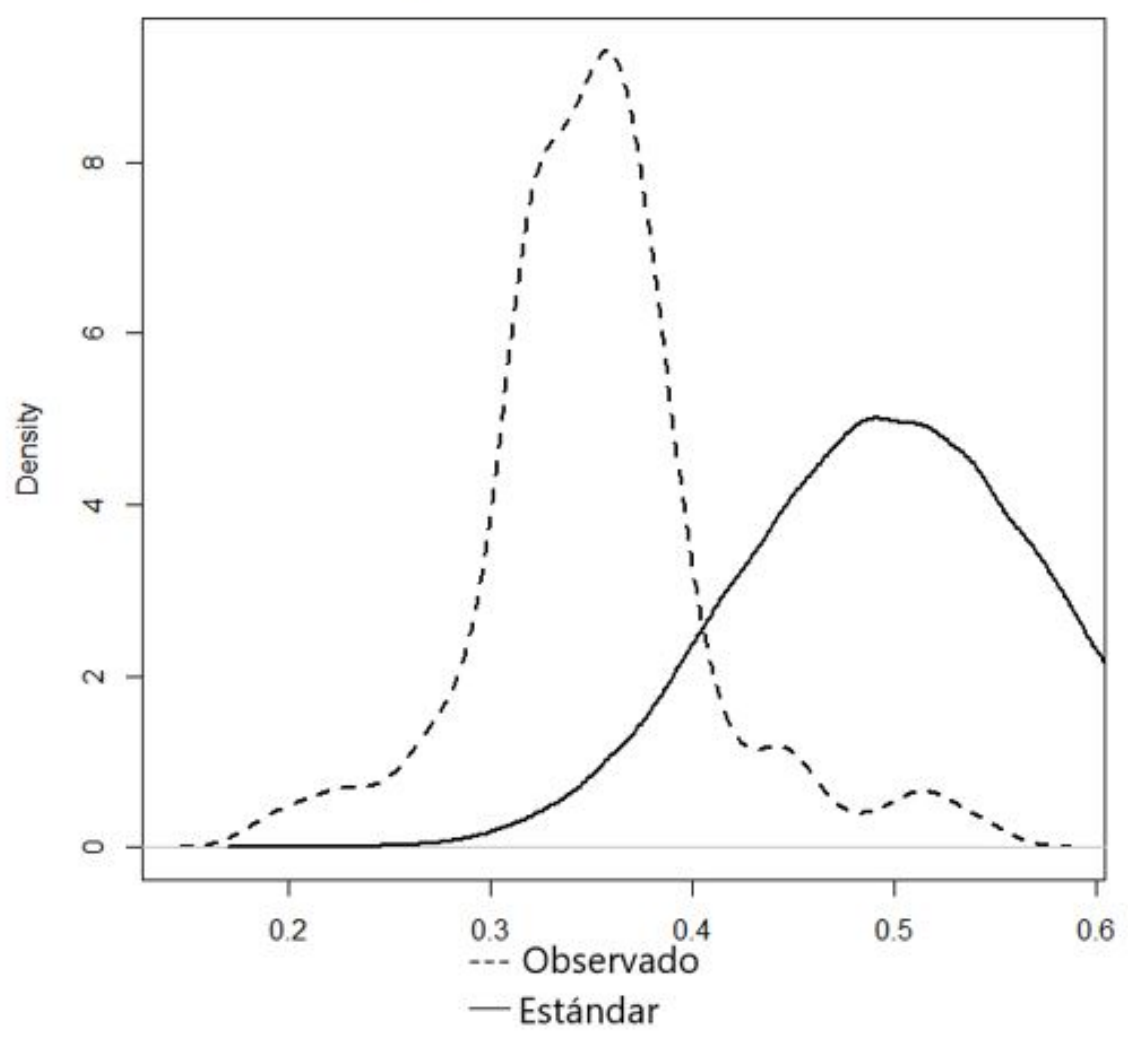

FIGURA 1.

Estimación de los datos.

Fuente: elaboración propia.

En la Figura 1, la curva punteada representa a los datos observados y la curva continua, el modelo teórico o de referencia. Se observa un fuerte distanciamiento entre las dos curvas, esto muestra las bajas puntuaciones de lo observado en relación a lo que se esperaría que fuera (curva continua). Se visualiza un fuerte apuntamiento en los valores observados para el índice de vigencia entre los valores 0.3 y 0.4 ; por tanto, es el primer cambio y desafío que plantea este análisis.

TABLA 3.

Momentos del modelo estándar y observado.

\begin{tabular}{l|l|l}
\hline Momento & Valor estándar & Valor observado \\
\hline Media & 0.5 & 0.34 \\
\hline Varianza & 0.0061 & 0.003 \\
\hline Simetria & 0 (Simétrica) & 0.46 (Asimetría positiva) \\
\hline Curtosis & 0.14 (Mesocurtica) & 2.13 (Leptocúrtica) \\
\hline
\end{tabular}

Fuente: elaboración propia.

En términos de centralidad y particularmente con el uso de la media, se visualiza que el comportamiento observado para el índice de vigencia es menor al estándar en prácticamente 0.15 unidades; en términos de variabilidad, con el uso de la varianza, se establece un comportamiento más homogéneo en el caso del índice de vigencia observado. Para el caso de los indicadores de forma, se observa un cambio en la asimetría y la curtosis, que pasa de un comportamiento simétrico para el modelo estándar a uno asimétrico positivo para el caso del índice de vigencia observado, esto significa que los datos tienden a agruparse en torno a valores 
pequeños. En relación a la curtosis, se pasa de un comportamiento mesocúrtico a leptocúrtico, reflejado en el fuerte apuntamiento del índice de vigencia observado (Tabla 3). Todas estas estadísticas permiten soportar el desafío para el índice de vigencia observado, pues se encuentra muy por debajo de lo que se espera en relación al modelo de referencia.

Si como proceso de acreditación institucional se establece, como uno de los criterios de decisión, que la probabilidad de obtener un índice de vigencia igual o superior a $60 \%$ debe ser mayor o igual a 0.50 . Como se conoce la distribución de probabilidad del índice de vigencia, la probabilidad solicitada es 0.36 , lo que significa que esta muestra observada de índices de vigencia da evidencia a favor del rechazo del cumplimiento de este requisito para la acreditación institucional, pues significa que se espera que el $36 \%$ de los planes formativos que constituyen las carreras pedagógicas de la universidad en cuestión deberían cumplir con tener un índice de vigencia superior al $60 \%$. Esto significa que se dispone de una herramienta cuantitativa para proyectar probabilidades de éxito o superación en función del índice de vigencia.

De manera similar se procederá a describir el índice DIAFORIN. En primer lugar, al representar las densidades para cada uno de los índices se visualiza una importante diferencia entre lo observado y lo estándar, como se señala en la Figura 2.

\section{Comparación Observado-Estándar}

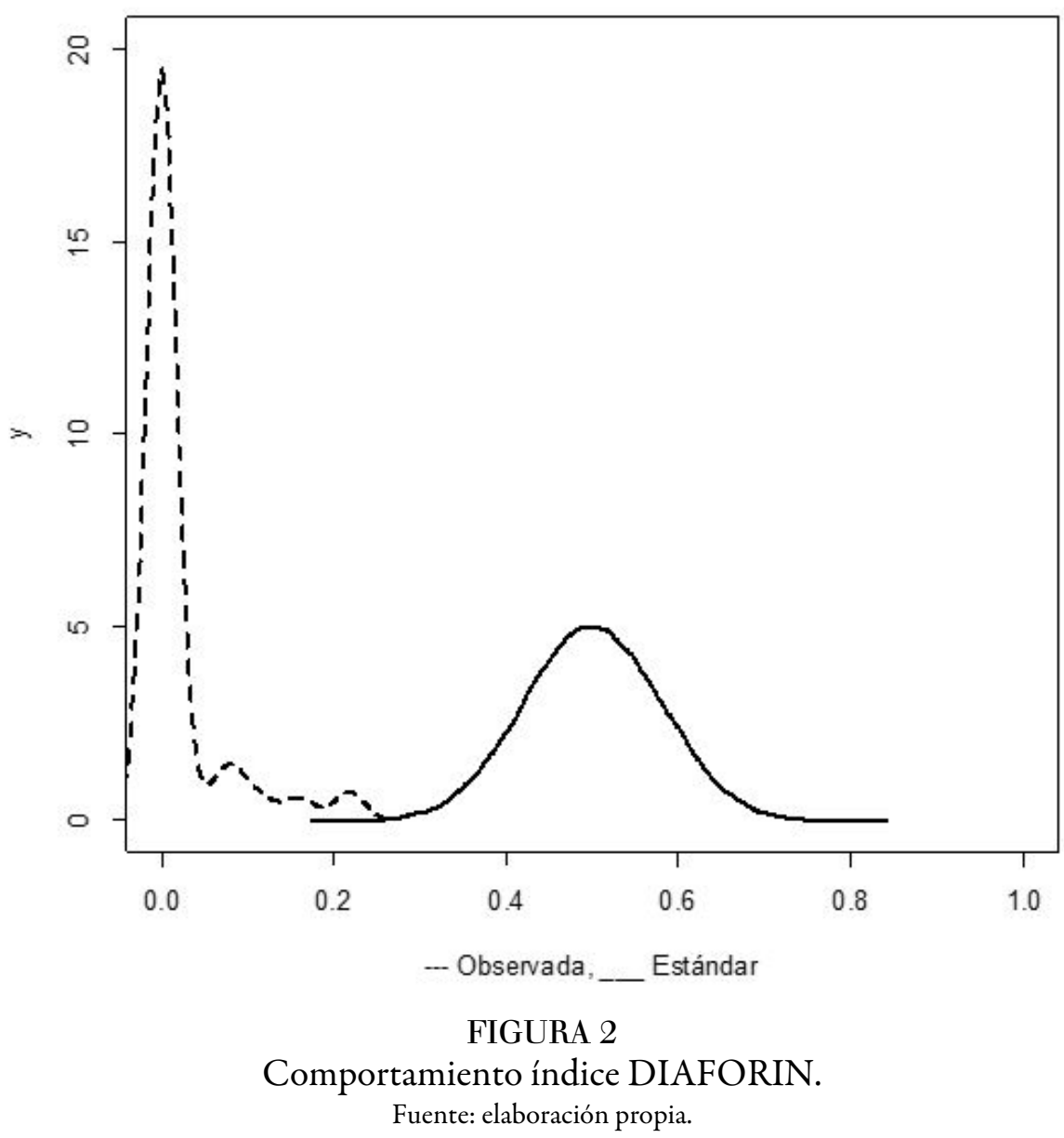

Como resultado del proceso de estimación asumiendo la distribución beta para estimar cambios, se obtiene que $\alpha=0.39$ y $\beta=5$.

Se observa un fuerte apuntamiento en los valores observados para el índice de DIAFORIN en torno a cero, este es el primer cambio y desafío que plantea este análisis. Esto significa que lo observado está muy por 
debajo de lo que se espera, lo que deja en evidencia objetiva el distanciamiento entre el conocimiento nuevo generado en la universidad en cuestión y la formación en esta perspectiva específica.

TABLA 4.

Momentos del modelo estándar y observado

\begin{tabular}{l|l|l}
\hline Momento & Valor estándar & Valor observado \\
\hline Media & 0.5 & 0.019 \\
\hline Varianza & 0.0061 & 0.002 \\
\hline Simetría & 0 (Simétrica) & 2.73 (Asimetría positiva) \\
\hline Curtosis & 0.14 (Mesocurtica) & 6.7 (Leptocúrtica) \\
\hline
\end{tabular}

Fuente: elaboración propia.

En la Tabla 4, en términos de centralidad y particularmente con el uso de la media, se visualiza que el comportamiento observado para el índice DIAFORIN es importantemente menor al estándar en prácticamente 0.48 unidades; en términos de variabilidad, con el uso la varianza, se establece un comportamiento más homogéneo en el caso del índice DIAFORIN observado. Para el caso de los indicadores de forma, se observa un cambio en la asimetría y la curtosis, que pasa de un comportamiento simétrico para el modelo estándar a uno asimétrico positivo para el caso del índice DIAFORIN observado; esto significa que los datos tienden a agruparse en torno a valores pequeños y de manera muy fuerte. En relación a la curtosis, se pasa de un comportamiento mesocúrtico a leptocúrtico, reflejado en el fuerte apuntamiento del Índice DIAFORIN observado.

Finalmente, en función de los valores de los índices, se proponen 5 categorías establecidas de manera clásica, es decir, generando particiones de igual amplitud, como se señalan en la Figura 3 para el índice de vigencia y en la Figura 4 para el índice DIAFORIN.

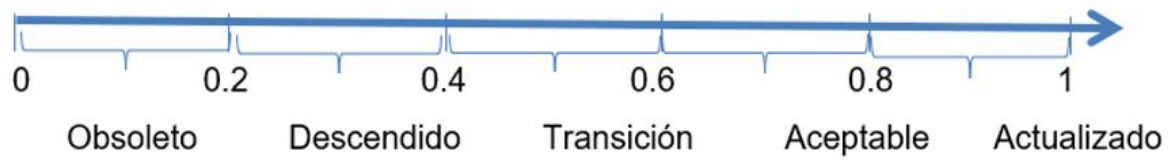

FIGURA 3.

Índice de vigencia.

Fuente: elaboración propia.

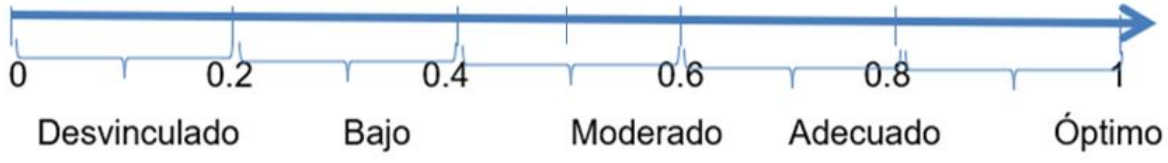

FIGURA 4.

Índice DIAFORIN.

Fuente: elaboración propia

En resumen, los índices definidos permiten incorporar un modelo de referencia o estándar. Además, permiten establecer una orientación y finalmente una categoría según el valor del índice obtenido, y es posible estimar probabilidades de transición, es decir, de pasar de una a categoría a otra. Por ejemplo, si se comparan dos universidades, es posible establecer probabilidades condicionales, esto es dado al encontrarse en la categoría Moderado, la cual es la probabilidad de pasar a la categoría Adecuado, por lo puede ser utilizada como una herramienta de priorización o comparación entre ambas instituciones. 
Disponer de este conjunto de herramientas cuantitativas permitirá dimensionar brechas, así como evaluar impactos de adecuaciones, disponer de criterios objetivos para la toma de decisiones, además de un abanico de opciones de comparación.

Finalmente, con base en la muestra observada, la Tabla 5 resume descriptivamente el índice de vigencia y DIAFORIN respectivamente.

TABLA 5.

Valores descriptivos de los índices de vigencia y DIAFORIN.

\begin{tabular}{lll}
\hline & Índice de vigencia & Índice de DIAFORIN \\
Media & 0.35 & 0.02 \\
Desviación estándar & 0.06 & 0.05 \\
Mínimo & 0.19 & 0.00 \\
Máximo & 0.54 & 0.22 \\
Asimetría & 0.46 & 2.80 \\
Curtosis & 2.13 & 7.21 \\
\hline
\end{tabular}

Fuente: elaboración propia.

En términos de centralidad y basado en la media, se puede establecer que el índice de vigencia identifica a una muestra en la categoría Descendido y para el caso del índice DIAFORIN en la categoría Desvinculado. En términos de variabilidad, se tienen comportamientos similares para ambos índices, donde son levemente más heterogéneas las puntuaciones para el índice de vigencia. En relación con el análisis de valores extremos, se observa que el índice DIAFORIN alcanza el menor de los mínimos, ya que este es de 0.00, mientras que el mayor de los máximos es obtenido por el índice de vigencia, que es de 0.54 . En función de la categorización para cada uno de los índices, esto significa que el máximo para el índice de vigencia es categorizado como en Transición, y el mínimo para el índice DIAFORIN como Desvinculado. Por otro lado, en términos de asimetría en ambos, se visualiza una asimetría positiva, esto significa que los datos observados tienden a agruparse en torno a valores pequeños, similarmente en curtosis, donde ambos índices reportan un comportamiento leptocúrtico, lo que se confirma con el apuntamiento de las curvas.

A continuación, en función de la evidencia emanada de los datos, se soportan las siguientes decisiones en relación a las hipótesis:

(1) En primer lugar, para decidir en relación a la hipótesis de que el índice DIAFORIN es significativamente mayor que cero $(\alpha=0.05)$, fue necesario verificar el supuesto de normalidad, para ello se utilizó la estadística de Shapiro-Wilk, supuesto que no fue cumplido ( $\mathrm{p}$-valor $=0.00$ ); por tanto, la prueba para una muestra se desarrolló con la estadística de Wilcoxon, con la que se obtuvo que el índice de DIAFORIN no es significativamente mayor que cero ( $\mathrm{p}$-valor $=0.32$ ), con lo cual no se rechaza esta hipótesis de investigación. Lo anterior significa que los datos soportan evidencia a favor de la nula relación entre la producción científica generada al interior de la universidad en cuestión y la formación en carreras pedagógicas.

(2) Similarmente, para la hipótesis referida a que el índice de conocimiento estático es significativamente mayor que el índice de conocimiento dinámico $(\alpha=0.05)$, también es rechazada la normalidad ( $\mathrm{p}$-valor $=0.00$ ); se justifica el uso de estadísticas no paramétricas como Wilcoxon. Se concluye que el índice de conocimiento estático es significativamente mayor que el índice de conocimiento dinámico; por tanto, esta hipótesis de investigación no es rechazada ( $\mathrm{p}$-valor $=0.00$ ). Esto significa que la presencia de libros como parte de las referencias bibliográficas de programas formativos se sobreponen a la presencia de artículos científicos. 
(3) Por último, se ha comprobado de manera teórica que no existe un estándar referencial para guiar la estructuración de un programa formativo, es decir, no existe un índice que guíe la construcción o reformulación de los programas de estudios de las universidades; por tanto, esta hipótesis no es rechazada.

Finalmente, se estimaron las probabilidades condicionales, dado que la muestra observada se encuentra en la categoría Descendido y Desvinculado para los índices de vigencia y DIAFORIN, respectivamente, para que se produzca un cambio de estado (avance) a Aceptable y Adecuado, respectivamente. Simbólicamente se tienen:

$\mathrm{P}$ (Aceptable

$\mathrm{P}$ (Adecuado $\mid$ Desvinculado $)=0.00$

Estas estimaciones dejan en evidencia lo difícil que es acceder a este nivel en función de los datos observados, de manera que refleja un gran desafío para la institución de poder avanzar en estas brechas y, definitivamente, es un llamado de atención con urgencia. De acuerdo con lo revisado en el marco teórico de este texto, la investigación y la articulación entre la teoría y la práctica siempre deben de estar presentes en todos los programas de estudio; adicionalmente, las universidades con su rol de aporte a la comunidad y al país deben promover las actividades académicas y de investigación. Una forma de verificar que esto se cumpla podría ser por medio de los índices que aquí se presentan.

\section{CONCLUSIONES}

Los resultados de este estudio han señalado que existe una baja representatividad (contribución) de la investigación que genera la propia universidad en los programas de estudios revisados. Lo anterior podría tener consecuencias directas en la formación de profesionales, ya que existen numerosas evidencias que la investigación contribuye a la formación de mejores profesionales, y en Educación no es la excepción. La literatura existente señala que las prácticas de investigación deben ser parte de la formación inicial docente, no para transformar a los futuros profesores y profesoras en personas expertas en investigación, sino para utilizar estas herramientas para potenciar sus propias prácticas pedagógicas, los procesos de aprendizaje-enseñanza y, especialmente, contribuir a mejorar día a día la educación del país.

Por otro lado, contar con índices de medidas como DIAFORIN (Diálogo entre Formación e Investigación), que den cuenta del diálogo entre la formación y la investigación, permitiría a las universidades cuantificar el grado de este vínculo al definir incrementos o variaciones y disponer de un soporte formal para la revisión constante de los programas. Paralelamente, el índice de vigencia permitiría evaluar continuamente la actualización e internacionalización de los programas de estudio, de esta manera se asegura un reajuste constante acorde a las necesidades actuales.

Así, se propone que en los programas de estudio de las carreras de pedagogía debe ser visible la contribución de la investigación, además de una bibliografía diversa. Por lo que se espera que los programas se encuentren en la categoría de vigencia como Aceptable y DIAFORIN como Adecuado, para asegurar una contribución mayor al $60 \%$.

Finalmente, es posible concluir que disponer de estas herramientas, índice de vigencia y DIAFORIN, permitiría a las instituciones de educación superior cuantificar el impacto de la investigación que produce la propia institución, registrar el aumento o disminución de este aporte en un determinado periodo de tiempo y, finalmente, contar con una actualización constante de las personas profesionales que se están formando.

\section{RECOMENDACIONES}

La toma de decisiones basadas en datos, los procesos empíricos y la disminución de la brecha entre investigación y el uso de este conocimiento deben ser procesos sistemáticos y planificados por las entidades 
responsables. El uso de índices, como los presentados en este estudio, podría ser un aporte a esta planificación y optimizaría los recursos, ya que, en general, el personal docente invierte gran parte de su tiempo en la producción de sus investigaciones y las universidades en recursos económicos incentivadores, pero es muy poco lo que se invierte en la difusión de la investigación a la comunidad educativa, su posterior capacitación y evaluación de uso, por ejemplo: en los programas formativos.

Por otro lado, sería interesante realizar jornadas de socialización, como seminarios u otras generadas por la universidad para dar a conocer estos resultados a otras unidades académicas y universidades de la región.

\section{Agradecimientos}

Agradecimiento y financiamiento

El autor José González agradece a la Universidad de Playa Ancha y el apoyo del Ministerio de Educación a través del Plan de Fortalecimiento de Universidades Estatales, UPA 1799.

\section{RefERENCIAS BIBLIOGRÁFICAS}

Aneas, A. y Vila, R. (2018). Entornos de desarrollo y aplicación de las competencias en el Prácticum de grado en Pedagogía de la Universidad de Barcelona. Revista Practicum, 3(1), 1-19.

Balladares-Burgos, J. (2018). Diseño pedagógico de la educación digital para la formación del profesorado. Revista Latinoamericana de Tecnología Educativa, 17(1), 41-60.

Boudon, R. y Lazarsfeld, P. (1973). De los conceptos a los indices empiricos. Metodología de las ciencias sociales. Laila.

Briceño, M., Correa, S., Valdés, M. y Hadweh, M. (2020). Modelo de gestión educativa para programas en modalidad virtual de aprendizaje. Revista de Ciencias Sociales, 26(2), 286-298.

Camarena, M. y Saavedra, M. (2018). La perspectiva de género en los programas de estudio de las licenciaturas contables administrativas. Revista de Ciencias Sociales y Humanidades, 27(54), 39-28.

Díaz, A. (2014). Construcción de programas de estudio en la perspectiva del enfoque de desarrollo de competencias. Perfiles Educativos, 36(143), 142-162.

Erraéz-Alvarado, J., Lucas-Aguilar, G., Guamán-Gómez, V. y Espinoza Freire, E. (2020). La investigación en estudiantes y docentes de la carrera de Educación Básica de la Universidad Técnica de Machala. Revista Conrado, 16(72), 163-170.

Grossman, P. y McDonald, M. (2008). Back to the future: Directions for research in teaching and teacher education. American Educational Research Journal, 45(1), 184-205.

Martí, J., Licandro, O. y Gaete, R. (2018). La Responsabilidad Social de la Educación Superior como Bien Común. Concepto y desafíos. Revista de Educación Superior, 47(186), 1-22.

Medina, J. y González, J. (2019). Graduation Profiles of Pedagogy Programmes According to the Current Educational Policies. International Education Studies, 12(6), 83-93.

Medina, J. y González, J. (2020). Índice estocástico de percepción del logro del perfil de egreso de estudiantes de pedagogía. El caso de una universidad regional de Chile. Formación Universitaria, 13(1), 83-92.

Merterns, L. (1997), “DACUM (desarrollo de un curriculum) y sus variantes SCID y AMOD”. Conocer.

Namakforoosh, M. (2000). Metodología de la investigación. Editorial Limusa.

Perines, H. (2017). Movilización del conocimiento en educación. Conexión entre la investigación, la política y la práctica: una aproximación teórica. Revista páginas de Educación, 10(1), 137-150.

Perines, H. (2018). ¿Por qué la investigación educativa no impacta en la práctica docente? Estudios sobre Educación, $34,9-27$.

Perines, H. y Campaña, K. (2019). La alfabetización de los futuros docentes en investigación educativa: Una reflexión teórica desde el contexto de Chile. RECETA. Revista Caribeña De Investigación Educativa, (1), 7-18. 
Quintana, M., Molina, J., Del Pozo, C., Díaz, L., Pérez, L. y Vingut, J. (2013). Consideraciones acerca del análisis del programa de estudios en el ejercicio pedagógico de promoción de categoría docente. Revista Cubana de Educación Médica Superior, 27(3), 255-261.

Quintero, J., Munévar, R. y Munevar, F. (2008). Semilleros de Investigación: Una estrategia para la formación de Investigadores. Educ.Educ, 11(1), 31-42.

Restrepo, B. (2003). Aportes de la investigación-acción educativa a la hipótesis del maestro investigador: evidencias y obstáculos. Educación y Educadores, 6, 91-104

Rodríguez, M., Alcázar, O. y Gil, S. (2019). Efectos de la aplicación de una estrategia pedagógica semilleros de investigadores en el desarrollo de las competencias investigativas en alumnos de pregrado de una universidad peruana (capítulo 4). En T. Sola, M. García, A. Fuentes, A Rodríguez y J. López (Eds.), Innovación Educativa en la Sociedad Digital (pp. 55-65). Editorial DYKINSON.

Tejeda, R. (2015). El aporte teórico en investigaciones asociadas a las ciencias pedagógicas.Didasc@lia: Didáctica y Educación, 6(6), 1-20.

UNESCO (2015). Replantear la educación. ¿Hacia un bien común mundial? UNESCO.

\section{Anexos}

\section{Anexo 1. Especificación de la definición de los indicadores basales}

Indicadores de internacionalidad ( ): Dice relación de cuántas de las referencias bibliográficas presentes en los programas formativos son en lengua anglosajona.

Actualidad ( ): Este indicador es función de la relación de distancia entre la referencia más antigua y la referencia más reciente. La relación funcional queda descrita por:

Contribución universitaria ( ): Este indicador dice relación de cuántas de las referencias son parte de los programas formativos o son producto de la misma universidad; estas pueden ser artículos, libros o tesis.

Conocimiento dinámico ( ): Este indicador dice la relación de cuántos de los referentes son artículos científicos.

Conocimiento estático ( ): Dice relación de cuántos de los referentes son libros.

Conocimiento gris ( ): Dice relación de cuántos de los referentes son documentos ministeriales o de gobierno, o de instituciones u organismos internacionales.

\section{Financiamiento}

Fuente: Universidad de Playa Ancha y Ministerio de Educación a través del Plan de Fortalecimiento de Universidades Estatales, UPA 1799.

No de contrato: 0546/2019.

Beneficiario: José González Campos

\section{INFORMACIÓN ADICIONAL}

Cómo citar: González-Campos, J. A. y Medina Pérez, J. (2022). Investigación y formación: un diálogo necesario. Revista Educación, 46(1). http://doi.org/10.15517/revedu.v46i1.45411 